Column Editor - Debra Paul, OTR/L

Getting through a traumatic experience is something that takes hold of one's life. Eleni's experience in the NICU ignited a passion within her and her family has followed in her footsteps. We are very appreciative to parents that take a leadership role in enhancing the lives of infants who are sick or who are born prematurely. Support from parents that have gone through a similar experience is invaluable and helps families navigate through what is often one of the most challenging times in their lives.

\title{
A Miraculous Journey
}

By Eleni Gerassis

DOI: $10.14434 /$ do.v14i2.32999

In: would like to share my miraculous story with you. While it was a nightmare experience, it is a story with a happy ending that has changed our lives for the better. My husband and I appreciate how blessed we are and are determined to help make a difference in the most crucial beginning of a baby's life.

Life was perfect. Mario and I had been married for three years with our beautiful little two-year-old Sophia. We were so happy to find out we were expecting our second child. At the 20-week ultrasound, we were told everything was normal and that we were having a little sister for Sophia, something I always dreamt about. We named her Dimitra (Dimi).

At just 24 weeks pregnant my dream turned into a living nightmare. I knew something was wrong. I was so sick, not able to move, and my baby wasn't moving. After extensive tests and consultations an initial diagnosis of Congenital Cystic Adenomatoid Malformation or otherwise known as CCAM, was made. CCAM is a rare abnormality in an infant's lung development and the medical team predicted a 10\% chance of survival for Dimi. For us it was a chance worth taking. We were rushed to the Obstetric Hospital where I stayed for one month on complete bed rest to prolong Dimi's birth and give her that extra chance to survive. Upon arrival to the hospital, both my unborn baby and I required special surgeries to drain large volumes of fluid from our bodies. I also had a rare in utero procedure that involved placing a shunt (drain) into the cyst growing inside Dimi's chest that would be removed during surgery after her birth.

At 30 weeks, I gave birth at Westmead Hospital. It was Mother's Day 2008 and a Mother's Day I will never forget. It was not your normal natural delivery. The room was filled with doctors and nurses explaining to us the possibility our baby might not survive and that she would be taken from us at once following the birth for urgent assistance. When Dimi was born, she was not breathing. Once she was stabilized, Dimi was placed on a ventilator and later transferred to the Grace Centre in preparation for surgery. We prepared for the worst and cried enough tears to fill a dam! Our supportive family, who we are so grateful for, stepped in with love and around-the-clock care for our two-year-old, Sophia, allowing Mario to constantly stay by my side in hospital. Four days after Dimi's surgery, her surgeon, the wonderful Dr. Soundappan, confirmed the growth in Dimi's

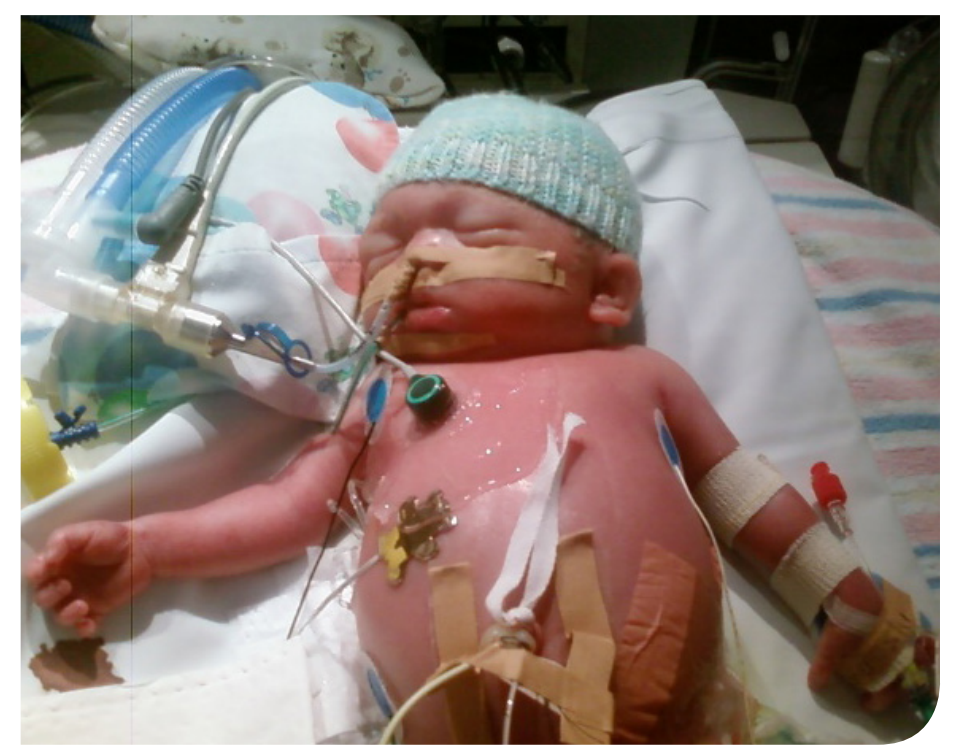

Dimi following surgery

chest was a benign Teratoma tumour and that all $500 \mathrm{~g}$ of it had been successfully removed from her tiny body. Dimi's weight was now approx. $1.5 \mathrm{~kg}$ and Mario's wedding band fit around her ankle.

Unfortunately, I did not have the pleasure of holding my Dimi for weeks and touched her through holes in her incubator. When I finally did get to hold Dimi, she was attached to machines. I felt robbed. I didn't want photos, gifts or anything that would remind me of her as I was so worried we would lose her. This was my way of dealing with things at the time. No beautiful flowers or congratulations. No going home with our baby. My time was filled with spending the days with her, and then having to leave her every night. Leaving her was absolute torture, even though we knew she was in the best hands.

Dimi spent the next four months in the Newborn Intensive Care Unit attached to a special machine to help her breathe and a feeding tube to help her grow enough to go home which she did. Our precious Dimi came home with us on Father's Day.

I thank God every day for blessing me with the most supportive husband who not once left my side and my two precious daughters, Sophia and Dimitra who everyday remind me about the true meaning of life! Dimi, I am convinced you were brought 


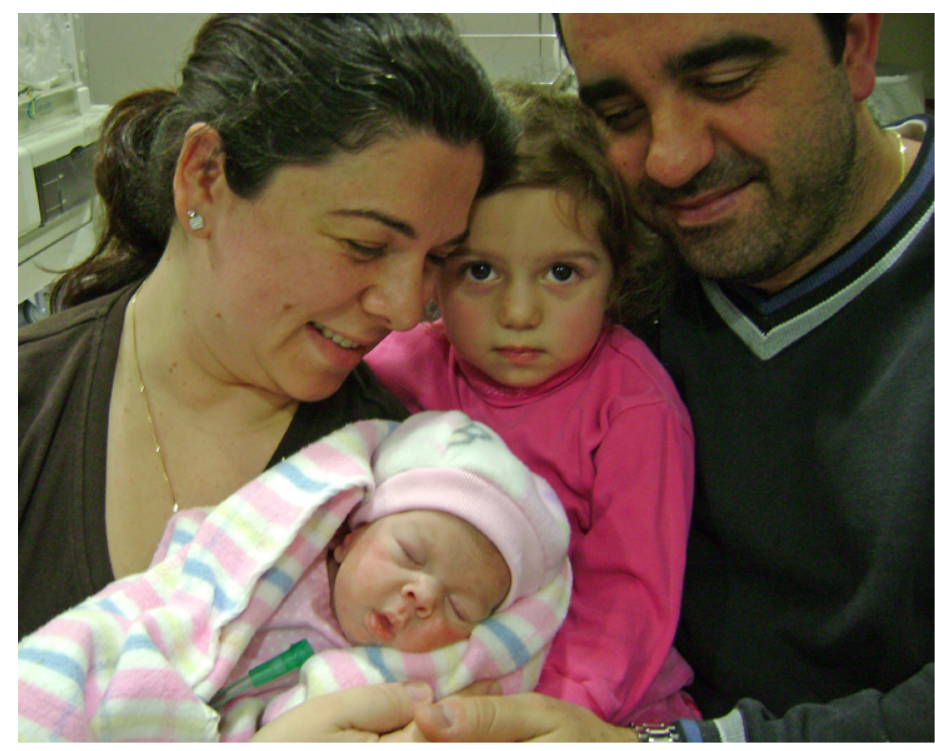

Eleni, Sophia and Mario enjoying the much-awaited time for a cuddle.

on this earth for a reason...to show us all to be fighters and to never give up. Thank you, Dimi, for making me the person I am today.

My husband and I made a promise to ourselves and our little Dimi that we would do everything possible to give back to the amazing work that saves critically ill newborn babies lives daily. We will be eternally grateful to the Grace Centre for Newborn Intensive Care at The Children's Hospital at Westmead for the lifesaving care our daughter received. The dedicated professional team at Grace Centre go above and beyond their call of duty, not only caring for critically ill newborn babies, but also supporting the families during the most traumatic experience of their lives. My family and I have supported this amazing facility over the past 10 years raising over $\$ 250,000$, purchasing lifesaving medical equipment, renovating various rooms within the unit, and contributing startup costs towards the Australasian NIDCAP Training Centre within Grace. After many years of fundraising for this cause so close to my heart, two years ago I was honored to be invited to join the Board of Directors of NIDCAP Australia. Through the many initiatives of the Australasian NIDCAP Training Centre, I can see how the programs are helping the babies and their parents at the Grace Centre.

Dimi often tells me when we are organizing our fundraising initiatives, 'Mum, I really love getting involved, it makes me happy, and I really care about the sick babies.' Hearing this warms my heart and reinforces to me how important it is that we appreciate all that we have and what we have been given. It also reminds me that I do what I do to help make that little bit of a difference for other families going through one of the most terrifying experiences one could ever go through whilst also supporting an amazing facility that cares for the country's sickest babies on a daily basis. Neonatal Intensive Care Units would not exist without the experienced, dedicated and highly trained staff that are required to care for our critically ill babies.

Dimi is now a healthy and bright 13-year-old in year 7 and absolutely loving high school. She is doing extremely well in all her subjects and tells us how one day she would love to be

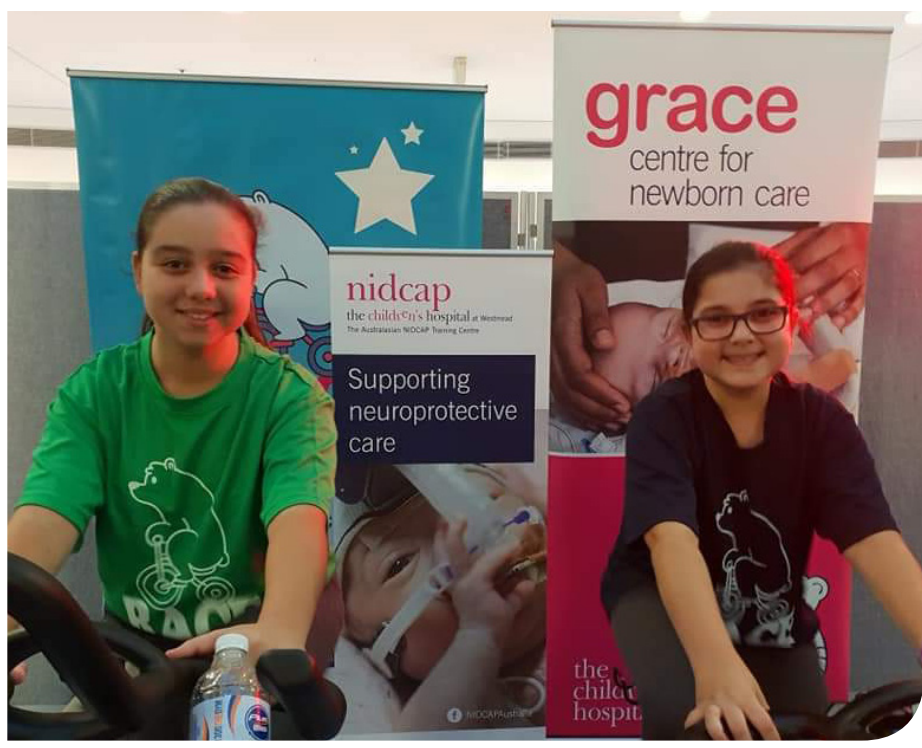

Dimi (right) with her sister Sophia participating in the fundraiser Race for Grace Bike Marathon

a "baby nurse". We are so proud of the young woman Dimi is growing up to be. She is a caring individual with a compassionate nature and determination to do well. One of most emotional and proudest moments for all of us was at the end of Dimi's last year at primary school. She became student leader, and at the year-end Award's ceremony Dimi received the one and only Citizenship Award. This award was given to a child who has always displayed continuous service to the school community, always puts others before herself, and is an active member in the school community.

I am proud to say both my girls are always the first to put their hands up when it comes to taking part in any school or community fundraising events or even just to simply lend a helping hand to their friends and family.

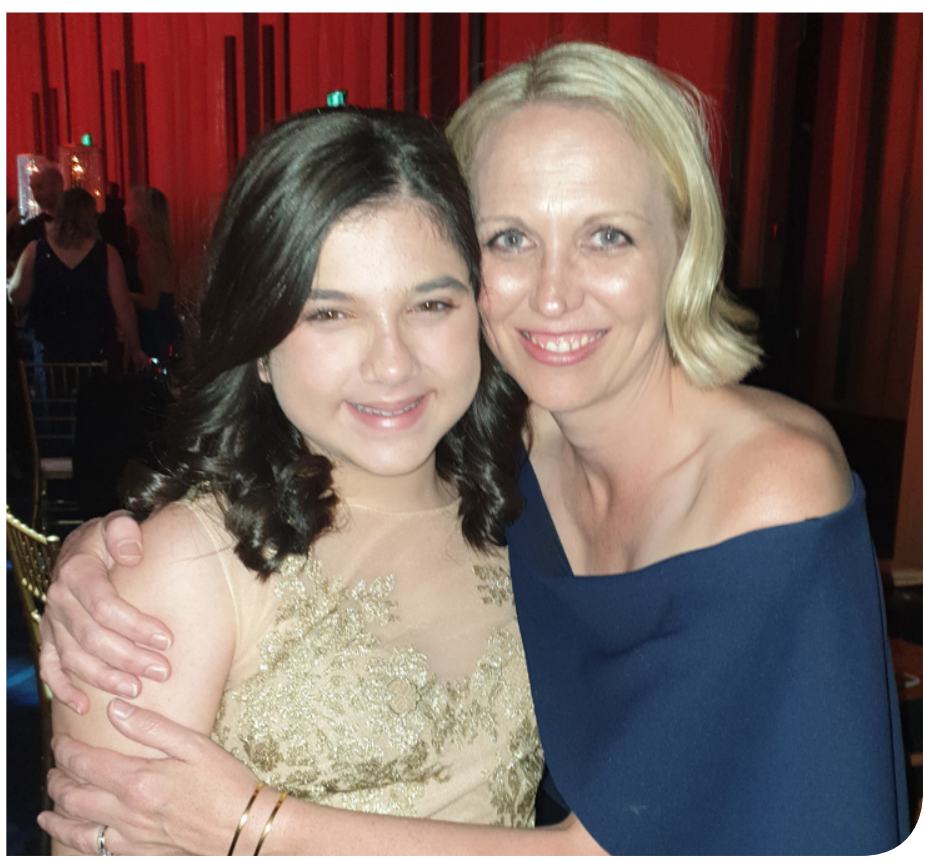

A special bond formed between Dimi and Kristen James Nunez, Nurse Practitioner and NIDCAP Professional. Photo taken at the Annual Grace Gala event 2021. 